\title{
REVIEW
}

\section{IS GO THE NEXT HUMAN BASTION TO CRUMBLE?}

\author{
COMPUTERS AND GAMES \\ Revised Selected papers of the $7^{\text {th }}$ International Conference, CG2010 \\ Kanazawa, Japan, Sep 2010 \\ H. Jaap van den Herik, Hiroyuki Iida \& Aske Plaat (Eds.) \\ Springer-Verlag 2011 \\ ISBN-10 3-642-17927-4 \\ $275 \mathrm{pp}$ \\ Reviewed by Dap Hartmann
}

This is the seventh in the series of Computers and Games conferences that are held every two years. The 2010 conference took place in Kanazawa, Japan, in September 2010. It was the third time that Japan organized this important meeting where computer games researchers from all over the world get together to discuss the latest developments.

The editors are very confident about the quality of the papers in this book: "The contributions to the Kanazawa conference showed considerable progress in the development and implementation of new ideas. Without any doubt, the quality of the papers of this conference coincides with the increase of the playing strength as appeared in many games." That is a bit of a peculiar statement because it suggests causality. The playing strength of computers increases because of better programs and faster hardware. It is difficult enough to separate one from the other, unless one carries out time-consuming experiments in which different versions of a program are tested on identical hardware, and vice versa, running identical versions of a program on different hardware. Even if the outcome of such experiments would prove that the software had improved regardless of the hardware speedup, it is still a big leap to link that to the quality of the papers presented at this conference. For one thing, maybe the best ideas are kept secret to maintain a competitive advantage?

The book contains 24 papers on a wide variety of subjects in no less than 15 different games. The classical games are rather underrepresented, with no papers on Chess, Checkers or Draughts. And there is not a single contribution on any card game. Most prominently represented among the classic games is Go. Major improvements have been made in recent years, and that seems to fuel the interest in what may well be the next human bastion to crumble. Go was long regarded too complex to conquer by computers because a 19x19 Go game tree of just the first six plies has more than 2 quadrillion $\left(=2\right.$ million billion $\left.=2 \times 10^{15}\right)$ leaf nodes (not accounting for transpositions). And with an average game length of about 300 plies, looking ahead a mere six plies is highly insignificant. However, with the application of non GOFAI (Good Old Fashioned Artificial Intelligence) techniques such as Monte Carlo simulations, the progress in playing strength has been quite spectacular. On the KGS Go server (www.gokgs.com), one of the largest Go servers in the world with more than 1500 people playing at any given time, ZEN, a Go program written by Ojima Yoji, has recently (May 2011) obtained the rank of 5 dan $^{1}$. To get a feeling for how strong that is: according to the European Go Database, there are only 7 players in France (population 66 million) that are higher ranked than ZEN. In the Netherlands (pop. 17 million) only 11 players, and in Germany (pop. 82 million) just 16 players are ranked above 5 dan. The European Go Federation (EGF) has developed a ranking system that converts the kye/dan classification into a rating system comparable to the Elo rating system. In that system, 5 dan corresponds to 2500 EFG. In April 2007, ZEN was only ranked at 2 kye (1900 EFG). If that rate of progress in playing strength continues, ZEN should play at professional level within a few years.

Kanazawa was not only hosting the CG2010 conference, also the $18^{\text {th }}$ World Computer Chess Championship and the $10^{\text {th }}$ Computer Olympiad took place at the same time. No real surprise at the WCCC when RYBKA won for the fourth consecutive time, well ahead of the competition. However, the Go 19x19 tournament at the Olympiad had a surprise winner. Not the 2008 Olympic Champion ZEN won this tournament, but the relatively unknown program ERICA by Shih-Chieh Huang, supervised by Rémi Coulomb took home the gold medal (after

\footnotetext{
${ }^{1}$ CrazyStone by Rémi Coulomb also has a 5 dan ranking on KGS and obtained that a month before ZEN. However, I could not find a historical record of CRAZYSTONE's progress over time. Furthermore, according to senseis.xmp.net: "In 2011-04 [...] CrazyStone reached solid 5d and held it for a short time. It quit playing games and its rating drifted down to 4d. [It] is holding a 4d rating as of 2011-05." For this reason, I discuss the progress of ZEN rather than CrazyStone.
} 
a playoff). Coulomb is associate professor of computer science at Université de Lille in France. He has been working on various computer games including Chess, Reversi, Othello, and Go. His chess program THE CRAZY BISHOP famously finished last-but-one in major computer chess tournaments. However, his Go program CRAZYSTONE won the 19x19 silver medal at the Computer Olympiad in Amsterdam in 2007. And now in Kanazawa, Coulomb was the advisor to gold medal winner ERICA. But most importantly, Rémi Coulomb also publishes regularly about computer Go. The paper he submitted to the CG2006 conference describes how CRAZYSTONE works. He even posted a Japanese translation of that paper on his website. For the present conference, he submitted a paper which was co-authored by Shih-Chieh Huang (programmer of ERICA) and Shuh-Shii Lin, both from National Taiwan Normal University. The paper describes 'Simulation Balancing', a new technique to tune the parameters of the Monte-Carlo playout method used in ERICA. The rate of success was measured by playing 9x9 games against FuEGO, a Canadian Go program by Enzenberger et al. Simulation Balancing improved ERICA's winning rate from $69 \%$ to $78 \%$. Nevertheless, FUEGO won the silver medal in the 9x9 tournament in Kanazawa, while ERICA finished in third place.

Monte Carlo is still a hot topic with no less than five papers containing this term in the title. After the successful application in Go, Monte-Carlo Tree -Search (MCTS) techniques are now also applied in other games such as LOA and Amazons. Julien Kloetzer from the Japan Advanced Institute of Science and Technology applied MCTS techniques to create opening books for Amazons. He noted that Amazons programs that use MCTS have difficulties using opening books that were created using minimax-based algorithms. While using MCTS to create opening books for Amazons does improve the quality of play, Kloetzer discovered along the way that Upper Confidence boundary Tree search (UCT) works even better. The best opening book improved the performance of his Amazons program CAMPYA by 45 Elo points. There is still a lot of work to be done as witnessed by CAMPYA's third place (out of four contestants) in the Amazons tournament during the Olympiad in Kanazawa. CAMPYA won only the four games it played against Martin Müller's ARROws2, which did not win a single game.

The paper "Solving Hex: Beyond Humans" by Arneson, Hayward and Henderson received the Best Paper Award at the conference. I do not know what the criteria were, but I have the feeling that the results (which are self-proclaimed to be a milestone) reported in this paper (automated Hex solvers can now solve many 9x9 Hex openings, thereby surpassing humans) had more impact than the quality of the paper itself. It is a good paper, no doubt about it, but is it really the best paper in this book? Other strong contenders in my humble opinion would have been "Improving Monte-Carlo Tree Search in Havannah" (and not just for the catchy title) by Richard Lorentz, "On the Scalability of Parallel UCT" by Richard Segal, and "New Solutions for Synchronized Domineering” by Bahri and Kruskal.

If you are interested in purchasing this book, it pays to shop around on the internet. At the Springer-Verlag website you pay €46.00. Amazon.com charges \$72.00 (€50.41), at Amazon.co.uk you pay £41.99 (€48.42), while at Amazon.de it costs only €38.99. 